\title{
A single center experience of adrenalectomy for adrenal tumors
}

\author{
Seung Keon Shin, Jong-Min Park \\ Department of Surgery, National Medical Center, Seoul, Korea
}

Purpose: In this study, we reviewed 16 cases of adrenalectomy, focusing specifically on the surgical outcomes and clinical courses.

Methods: The data from 16 patients who underwent an adrenalectomy at our hospital between January 2007 to December 2016 were retrospectively analyzed based on their medical records. Data available for each patient included age, sex, tumor location, length of postoperative hospital stay, tumor size, tumor pathology, final diagnosis, operation time, operative blood loss, and type of operation.

Results: The study population consisted of eight males and eight females, with a mean age of 58.00 \pm 11.34 years (range, 33-76 years). The mean tumor size was $2.78 \pm 2.02 \mathrm{~cm}$ (range, $0.5-7 \mathrm{~cm}$ ). Fourteen patients were diagnosed with functional adrenal tumors and two with nonfunctional adrenal tumors. Compared to laparoscopic adrenalectomy, open surgery resulted in a statistically significantly longer operating time, increased operative blood loss, and a longer postoperative hospital stay.

Conclusion: If the hormonal activity of adrenal tumors is not confirmed, the tumor should be considered functional when accompanied by related symptoms and an adrenalectomy should be performed. In addition, we suggest that a laparoscopic adrenalectomy is superior to an open adrenalectomy in terms of recovery after surgery, despite the small number of cases evaluated. Open adrenalectomy should be considered following comprehensive consideration of the patient's condition, such as accompanying surgery or metastatic cancer.

Keywords: Adrenalectomy, Adrenal glands, Laparoscopy, Laparotomy

\section{INTRODUCTION}

Because of the small size and retroperitoneal location of the adrenal glands, adrenalectomy prior to the introduction of laparoscopic surgery required a large laparotomy. Since the first laparoscopic adrenalectomy was described by Gagner et al. [1] in 1992, laparoscopy has been widely used for adrenalectomy. In particular, for benign adrenal tumors, laparoscopic adrenalectomy is considered a priority as reduced blood loss, shorter hospital stays, and faster recovery times are expected, compared with open surgery [1-4].

Based on the above rationale, we have performed laparoscopic or open adrenalectomy for the past 10 years. In this study, we reviewed several cases of adrenalectomy, focusing on the surgical

Received: Oct 10, 2017 Accepted: Nov 1, 2017

Correspondence to: Jong-Min Park

Department of Surgery, National Medical Center, 245 Eulji-ro, Jung-gu, Seoul 04564, Korea

Tel: +82-2-2260-4883, Fax: +82-2-2269-0750

E-mail: jmparkgs@gmail.com

Copyright ( $($ Korean Society of Surgical Oncology

This is an Open Access article distributed under the terms of the Creative Commons Attribution Non-Commercial License (http://creativecommons.org/licenses/by-nc/4.0) which permits unrestricted non-commercial use, distribution, and reproduction in any medium, provided the original work is properly cited. outcomes and clinical course. We describe the demographic composition of the cases and our findings focused on three topics: (1) the relationship between the hormonal activity of the adrenal tumor and the indication for surgery, (2) comparison of the clinical outcomes between laparoscopic and open adrenalectomy, and (3) conditions that require an open adrenalectomy.

\section{METHODS}

All patients were informed about the procedure and consents for surgery were obtained. This study was approved by the Institutional Review Board of the National Medical Center.

The data from 16 patients who underwent an adrenalectomy at our hospital from January 2007 to December 2016, including age, sex, tumor location, postoperative hospital stay, tumor size, tumor pathology, final diagnosis, operation time, operative blood loss, and type of operation, were analyzed retrospectively. Operation time and operative blood loss were evaluated based on anesthesia records.

We performed an adrenalectomy based on several criteria at our hospital. First, if adrenal tumors were identified with accompanying symptoms such as hypertension using radiological examina- 
tion, hormonal activity was confirmed with hormonal work-ups and an adrenalectomy was performed. Second, an adrenalectomy was performed regardless of the hormonal work-up result if there were symptoms that affected daily life, such as worsening hypertension, and a confirmation of an adrenal tumor by radiologic examination. Third, adrenalectomy was performed when the size of adrenal tumor had substantially increased at follow-up. Fourth, malignant tumors, including metastatic cancer, were also surgically removed. Fifth, if abdominal surgery was performed for other reasons, known adrenal tumors were also removed simultaneously, even if they did not meet the above criteria. When taking all these criteria into consideration, the patient's general status and expected benefits from adrenalectomy were comprehensively considered.

A laparoscopic adrenalectomy was performed in all cases using the transperitoneal approach. Patient posture was changed to the left lateral position or right lateral position after systemic inhalation anesthesia in the supine position. There was no significant difference in the surgical procedure compared to previous studies $[5,6]$. In the right-side position, four trocars were used because liver traction was necessary. In the left-side position, only three trocars were used (Fig. 1). The position of the trocar was inserted variably depending on the anatomical structure of the patient. A flexible laparoscope was used alternately to secure a good visual field. The right adrenalectomy was performed by pulling the liver upward with a liver retractor, separating the liver triangular ligament, and then carefully removing the soft tissue between the inferior vena cava to allow full exposure of the adrenal gland. The adrenal vein was then ligated with a $5-\mathrm{mm}$ clip, and multiple adrenal arteries were carefully ligated. Left adrenalectomy was initiated by separating the left colon from the retroperitoneum and the spleen, and pulling the adrenal gland upwards. The adrenal vein directed

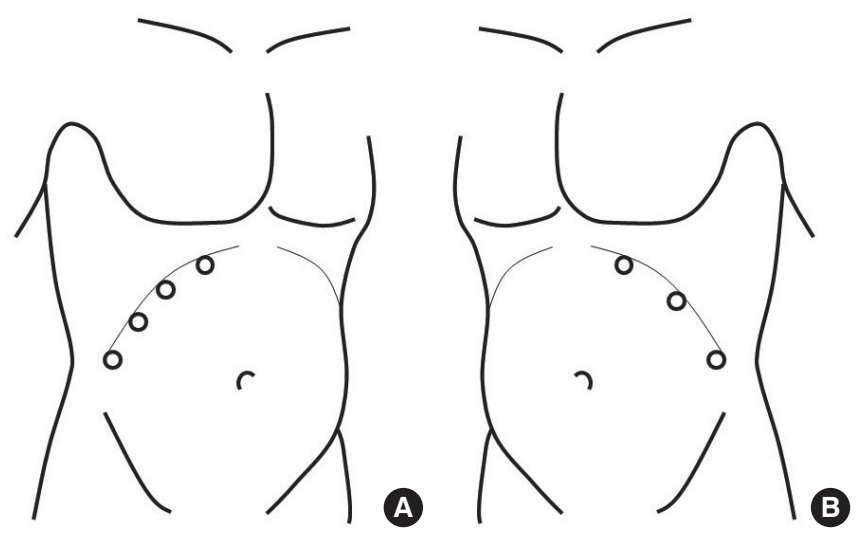

Fig. 1. Trocar placement is indicated by closed circles. Four trocars were used in case of the right laparoscopic adrenalectomy (A), and three trocars were used in case of the left adrenalectomy (B). to the left renal vein was ligated first. Subsequently, several adrenal artery branches were carefully ligated. Completely resected adrenal tumors were removed from the abdominal cavity along with the trocar after confirming that hemostasis was complete.

The patients were divided into two groups, those who underwent a laparoscopic adrenalectomy and those who underwent open surgery. The following outcomes were compared between the two groups: operation time, operative blood loss, and postoperative length of hospital stay. The independent t-test was used to examine group differences. P-values of $<0.05$ were considered statistically significant. SPSS ver. $18.0 \mathrm{~K}$ for MS Windows (IBM Corp., Armonk, NY, USA) was used for statistical analysis.

\section{RESULTS}

Sixteen patients (eight males and eight females) underwent an adrenalectomy. The demographic composition of patients is summarized in Table 1. The patients' mean age was $58.00 \pm 11.34$ years (range, 33-76 years). Three patients had undergone previous abdominal surgery; a 54-year-old female who underwent surgery due to an ectopic pregnancy 25 years ago, a 68-year-old female who underwent an appendectomy 50 years ago, and a 69-year-old female who underwent a hysterectomy with uterine leiomyoma 25 years ago. Regarding other comorbidities, 11 patients had hypertension, two of which had diabetes mellitus. Among patients with both hypertension and diabetes mellitus, one patient had a history of microvascular angina. Among the other patients with hyperten-

Table 1. Patient demographics

\begin{tabular}{lc}
\hline Characteristic & Value \\
\hline Age (yr) & $58.00 \pm 11.34$ \\
Mean & $33-76$ \\
Range & \\
Sex (n) & 8 \\
Male & 8 \\
Female & \\
Tumor location & 7 \\
Right & 9 \\
Left & 0 \\
Both & \\
Tumor size (cm) & $2.78 \pm 2.02$ \\
Mean & $0.5-7.0$ \\
Range & \\
Previous abdominal operation history & 3 \\
(+) & 13 \\
(-) & \\
Laparoscopic vs. open surgery & 12 \\
Laparoscopic & 4 \\
Open &
\end{tabular}


Table 2. Pathologic results and final diagnoses

\begin{tabular}{llc}
\hline Pathology & \multicolumn{1}{c}{ Final diagnosis } & No. \\
\hline Functioning adrenal tumor & & 14 \\
Aldosterone-producing adenoma & Primary aldosteronism & 8 \\
Cortisol-producing adenoma & Cushing's syndrome & 3 \\
Pheochromocytoma & Pheochromocytoma & 1 \\
Cortical adenoma & Cortical adenoma & 1 \\
Adrenal hyperplasia & Adrenal hyperplasia & 1 \\
Nonfunctional adrenal tumor & & 2 \\
Cortical adenoma & Cortical adenoma & 1 \\
Metastatic adenocarcinoma & Metastatic adenocarcinoma & 1 \\
Total & & 16 \\
\hline
\end{tabular}

sion, one patient had angina pectoris, one had unstable angina, one had hypertrophic cardiomyopathy, and one had both chronic renal failure and cerebral infarction. Regarding the location of the tumors, the tumor was positioned on the right adrenal gland in seven patients, and on the left adrenal gland in nine. None of the patients had tumors on both sides. The mean tumor size of was $2.78 \pm 2.02 \mathrm{~cm}$; the smallest tumor was $0.5 \mathrm{~cm}$ and the largest was 7 $\mathrm{cm}$. Twelve patients underwent a laparoscopic adrenalectomy, while four patients underwent open surgery. Initial laparoscopic adrenalectomy in a 49-year-old male patient was converted to open surgery during the operation because of the difficulty in obtaining the visual field. This patient was included with the open surgery cases.

Fourteen patients were diagnosed with hormonally active (functional) adrenal tumors. Of these, 12 cases were confirmed through hormonal work-ups. In the remaining two patients, hormonal activities were not evaluated, but they were diagnosed with functional adrenal tumors based on the clinical features, such as improvement of postoperative hypertension. Of the aldosterone-producing adenomas in eight patients, hypertension and hypokalemia were observed in seven patients and not in the other patient. Of the three patients with cortisol-producing adenomas, hypertension was observed in two. Hypertension was not observed in the other patient with cortisol-producing adenoma and one patient with pheochromocytoma. In the two patients with hormonally silent (nonfunctional) adrenal tumors, there were no accompanying symptoms such as hypertension. In addition, there was no record of hormonal work-ups, because one patient was a 70-year-old male with early gastric cancer who was scheduled to undergo a gastrectomy at the same time and the other was a 74-year-old male with metastatic adenocarcinoma metastasized from rectal cancer. Final diagnoses were based on the clinical symptoms and pathologic results. There were eight cases of primary aldosteronism, three cases of Cushing's syndrome, and two cases of cortical adenoma. The other cases were pheochromocytoma, adrenal hyper-
Table 3. Comparison of operative outcomes between laparoscopic and open surgery

\begin{tabular}{lccc}
\hline & $\begin{array}{c}\text { Laparoscopic } \\
(n=12)\end{array}$ & $\begin{array}{c}\text { Open } \\
(n=4)\end{array}$ & P-value ${ }^{\text {a) }}$ \\
\hline Operation time (min) & $121.75 \pm 67.54$ & $235.80 \pm 94.06$ & 0.006 \\
Operative blood loss $(\mathrm{mL})$ & $4.17 \pm 5.15$ & $30.00 \pm 12.25$ & 0.000 \\
Postoperative hospital stay (day) & $8.00 \pm 4.67$ & $22.40 \pm 12.58$ & 0.001 \\
\hline
\end{tabular}

Values are presented as mean \pm standard deviation.

a) P-values were computed by using the independent t-test. P-values $<0.05$ were considered statistically significant.

plasia, and metastatic adenocarcinoma (Table 2). Compared to laparoscopic adrenalectomy, open surgery resulted in a statistically significantly longer operating time $(\mathrm{P}=0.006)$, increased operative blood loss $(\mathrm{P}=0.000)$, and a longer postoperative hospital stay $(\mathrm{P}=0.001)($ Table 3$)$.

\section{DISCUSSION}

In this study, we asked three questions. First, when is the indication for surgery when not proven positive in the hormonal work-up? Second, does a laparoscopic adrenalectomy lead to a better postoperative recovery than an open adrenalectomy in the small number of cases examined? Third, what are the conditions that require an open adrenalectomy? In this section, we describe the implications of this study with these three perspectives.

According to the Guidelines issued by the American Association of Clinical Endocrinologists (AACE) and the American Association of Endocrine Surgeons (AAES) [7], one important factor in determining the treatment strategy for adrenal tumors is whether the tumors are functional. In general, a hormonal work-up is performed to determine whether adrenal tumors are functional. The AACE and AAES suggest the 2-day low-dose dexamethasone suppression test as a suitable hormonal work-up. In addition, the urinary free cortisol test, the urinary metanephrine-to-creatinine ratio, or the renin-to-aldosterone ratio are also considered useful tests. If a hormonal excess is observed, surgery is indicated for the adrenal tumor.

We hypothesized that an adrenalectomy could be considered if the symptoms associated with functional adrenal tumors are present, even if the adrenal tumor has not been determined functional on a hormonal work-up. As a result, if an adrenalectomy improves the clinical symptoms, the adrenal tumor may be regarded as functional. In our study, the hormonal work-up was negative in two patients with hypertension; however, we could not find any other cause of the hypertension, other than the adrenal tumor, thus we 
performed an adrenalectomy. The surgery resulted in excellent results that relieved the symptoms of hypertension. Based on this outcome, we diagnosed two patients with functional adrenal tumors.

Surgery is generally not indicated for nonfunctional adrenal tumors; however, we assumed that surgery can be performed depending on the patient's circumstances. In our study, two patients were diagnosed with nonfunctional adrenal tumors. A 70-year-old male with a left adrenal cortical adenoma did not have any significant symptoms associated with an adrenal tumor or a positive result hormonal work-up result; however, a scheduled gastrectomy for early gastric cancer was performed and the cortical adenoma could also be excised. In addition, a 74-year-old male underwent surgery for rectal cancer; despite having a nonfunctional adrenal tumor, an adrenal metastasis was suspected in a preoperative radiologic examination and the adrenal tumor was planned to be resected together. These two patients were diagnosed with nonfunctional tumors, but nevertheless had undergone an adrenalectomy. Therefore, in conclusion to our first question, even if an adrenal tumor is not confirmed as functional or nonfunctional, it may be desirable to perform an adrenalectomy if the adrenal tumor is the only cause of the related symptoms, such as worsening hypertension. Even in the case of a nonfunctional tumor, it is considered to be removed if there is another reason for resection, such as the differential diagnosis of metastatic cancer or the scope for accompanying surgery.

Regarding the surgical methods, laparoscopic adrenalectomy has been widely accepted since its first description and implementation as it has advantages over an open adrenalectomy. In particular, the superiority of the laparoscopic adrenalectomy in postoperative recovery has been confirmed in several studies $[6,8,9]$; a similar result was observed in our study. Compared to an open adrenalectomy, laparoscopic surgery resulted in a significantly shorter operating time, decreased operative blood loss, and a shorter postoperative hospital stay. As a result, regarding the second question on postoperative recovery, we can confirm that even in a small number of cases, the postoperative recovery after a laparoscopic adrenalectomy was superior to an open adrenalectomy.

Nevertheless, open surgery is strongly recommended if adrenal carcinoma is suspected or confirmed. Because malignant tumors are aggressive, open surgery is beneficial for en-bloc resection $[10,11]$. In fact, these criteria are now the subject of controversy, because several large-scale studies have shown that open surgery and laparoscopic adrenalectomy produce equivalent results [1214]. On the other hand, several previous studies recommend open surgery $[15,16]$, and in the 2013 guidelines published by the Society of American Gastrointestinal and Endoscopic Surgeons, open surgery is recommended until there is firm evidence confirming that laparoscopic adrenalectomy is more favorable for adrenal carcinoma [17]. A recent retrospective study of 201 patients in 13 tertiary care cancer centers concluded that a laparoscopic adrenalectomy was an acceptable option for adrenocortical carcinomas $<10$ $\mathrm{cm}$ in size [18]. However, in the same study, the authors had one prerequisite: if local invasion or enlarged lymph nodes were identified, open surgery should be chosen regardless of tumor size.

In another case, a metastatic adenocarcinoma was removed from a rectal cancer patient. Although recent studies have shown that a laparoscopic adrenalectomy can be performed even with a solitary adrenal metastasis, without evidence of additional metastasis $[19,20]$, metastatic adenocarcinoma was also removed using open surgery in our case because a lower anterior resection was performed simultaneously for rectal cancer. As a result, we achieved satisfactory results without any sequelae. Therefore, in answer to our third question, when deciding to perform an open adrenalectomy, the patient's condition, such as accompanying surgery or metastatic cancer, should be considered. As mentioned earlier, the criteria for open surgery are controversial; therefore, more extensive studies should be conducted to determine when to choose open surgery, despite the disadvantages of postoperative recovery.

In summary, we have identified the following three answers to the questions posed in this study. First, even when not proven positive in a hormonal work-up, an adrenalectomy may be desirable if the adrenal tumor is suspected to be symptomatic. Second, even in the small number of cases assessed in this study, the postoperative recovery after a laparoscopic adrenalectomy was confirmed to be superior to that after an open adrenalectomy. Third, open adrenalectomy should be considered following comprehensive consideration of the patient's condition, such as with accompanying surgery or in cases of metastatic cancer.

There are two key limitations to this study. First, the number of patients is relatively small to discuss the statistical significance meaningfully. Surgery on adrenal tumors is not performed frequently, therefore, studies in a single hospital are limited in size. In future studies, the surgical data from many hospitals should be utilized in an integrated and organic way. This will lead to more meaningful conclusions. We will continue to follow-up with more adrenalectomy cases too. Second, this study summarizes the experience of the first decade of adrenalectomy practice in our hospital. Considering together with the small number of cases discussed above, the initial insufficiency of experience could affect the surgeons' preference of the surgical method; however, this is also why we are reporting on our experiences. This study is valuable as it provides the basic data to investigate the accumulation of surgical 
experience in a follow-up study. If the surgical outcome of adrenalectomy procedures improves in our hospital in the future, this data will be valuable in identifying the cause for this.

\section{CONFLICT OF INTEREST}

No potential conflict of interest relevant to this article was reported.

\section{ACKNOWLEDGMENTS}

We would like to thank Junghun Kim, MD and Jin-woo Jun, MD for their comments that greatly improved the manuscript.

\section{REFERENCES}

1. Gagner M, Lacroix A, Bolte E. Laparoscopic adrenalectomy in Cushing's syndrome and pheochromocytoma. N Engl J Med 1992;327:1033.

2. Miccoli P, Raffaelli M, Berti P, Materazzi G, Massi M, Bernini G. Adrenal surgery before and after the introduction of laparoscopic adrenalectomy. Br J Surg 2002;89:779-82.

3. Ortega J, Sala C, Garcia S, Lledo S. Cost-effectiveness of laparoscopic vs open adrenalectomy: small savings in an expensive process. J Laparoendosc Adv Surg Tech A 2002;12:1-5.

4. Lee J, El-Tamer M, Schifftner T, Turrentine FE, Henderson WG, Khuri S, et al. Open and laparoscopic adrenalectomy: analysis of the National Surgical Quality Improvement Program. J Am Coll Surg 2008;206:953-9.

5. Duh QY, Siperstein AE, Clark OH, Schecter WP, Horn JK, Harrison MR, et al. Laparoscopic adrenalectomy. Comparison of the lateral and posterior approaches. Arch Surg 1996;131:870-5.

6. Fernandez-Cruz L, Saenz A, Benarroch G, Astudillo E, Taura P, Sabater L. Laparoscopic unilateral and bilateral adrenalectomy for Cushing's syndrome: transperitoneal and retroperitoneal approaches. Ann Surg 1996;224:727-34.

7. Zeiger MA, Thompson GB, Duh QY, Hamrahian AH, Angelos P, Elaraj D, et al. The American Association of Clinical Endocrinologists and American Association of Endocrine Surgeons medical guidelines for the management of adrenal incidentalomas. Endocr Pract 2009;15 Suppl 1:1-20.

8. Elfenbein DM, Scarborough JE, Speicher PJ, Scheri RP. Comparison of laparoscopic versus open adrenalectomy: results from
American College of Surgeons-National Surgery Quality Improvement Project. J Surg Res 2013;184:216-20.

9. Brunt LM, Doherty GM, Norton JA, Soper NJ, Quasebarth MA, Moley JF. Laparoscopic adrenalectomy compared to open adrenalectomy for benign adrenal neoplasms. J Am Coll Surg 1996;183:110.

10. Schulick RD, Brennan MF. Long-term survival after complete resection and repeat resection in patients with adrenocortical carcinoma. Ann Surg Oncol 1999;6:719-26.

11. Schulick RD, Brennan MF. Adrenocortical carcinoma. World J Urol 1999;17:26-34.

12. Palazzo FF, Sebag F, Sierra M, Ippolito G, Souteyrand P, Henry JF. Long-term outcome following laparoscopic adrenalectomy for large solid adrenal cortex tumors. World J Surg 2006;30:893-8.

13. Brix D, Allolio B, Fenske W, Agha A, Dralle H, Jurowich C, et al. Laparoscopic versus open adrenalectomy for adrenocortical carcinoma: surgical and oncologic outcome in 152 patients. Eur Urol 2010;58:609-15.

14. Porpiglia F, Fiori C, Daffara F, Zaggia B, Bollito E, Volante M, et al. Retrospective evaluation of the outcome of open versus laparoscopic adrenalectomy for stage I and II adrenocortical cancer. Eur Urol 2010;57:873-8.

15. Miller BS, Ammori JB, Gauger PG, Broome JT, Hammer GD, Doherty GM. Laparoscopic resection is inappropriate in patients with known or suspected adrenocortical carcinoma. World J Surg 2010;34:1380-5.

16. Gonzalez RJ, Shapiro S, Sarlis N, Vassilopoulou-Sellin R, Perrier ND, Evans DB, et al. Laparoscopic resection of adrenal cortical carcinoma: a cautionary note. Surgery 2005;138:1078-85.

17. Stefanidis D, Goldfarb M, Kercher KW, Hope WW, Richardson W, Fanelli RD, et al. SAGES guidelines for minimally invasive treatment of adrenal pathology. Surg Endosc 2013;27:3960-80.

18. Lee CW, Salem AI, Schneider DF, Leverson GE, Tran TB, Poultsides GA, et al. Minimally invasive resection of adrenocortical carcinoma: a multi-institutional study of 201 patients. J Gastrointest Surg 2017;21:352-62.

19. Moreno P, de la Quintana Basarrate A, Musholt TJ, Paunovic I, Puccini M, Vidal O, et al. Adrenalectomy for solid tumor metastases: results of a multicenter European study. Surgery 2013;154: 1215-22.

20. Wang DS, Terashi T. Laparoscopic adrenalectomy. Urol Clin North Am 2008;35:351-63. 